\title{
Helicobacter pylori eradication: A randomised comparative trial of 7-day versus 14-day triple therapy
}

\author{
Ahmed Sokwala, Mahesh V Shah, Smita Devani, Gerald Yonga
}

Background. Helicobacter pylori is associated with several upper gastrointestinal conditions including chronic gastritis, peptic ulcer disease, and gastric malignancy. Proton pump inhibitor-based triple therapies are considered the standard regimens for $H$. pylori eradication, but the optimal duration of therapy is controversial. To prevent infection and complications, local studies should be undertaken to evaluate $H$. pylori eradication rates in a country.

Objectives. We compared 7-day and 14-day regimens to determine the optimum duration of triple therapy for $H$. pylori eradication.

Methods. We undertook a prospective randomised comparative trial of 7-day and 14-day triple therapy regimen for $\mathrm{H}$. pylori eradication at the Aga Khan University Hospital, Nairobi; 120 patients with dyspepsia and $H$. pylori infection were randomised to receive esomeprazole, amoxicillin and clarithromycin for either 7 days (EAC 7) or 14 days (EAC 14). Compliance and side-effects were assessed 2 weeks after the start of therapy and $H$. pylori eradication was assessed by stool antigen tests 4 weeks after treatment.

Results. Both the intention-to-treat (ITT; $N=120$ ) and per protocol (PP; $N=97)$ analyses showed no significant differences between the eradication rates of EAC 7 (ITT 76.7\%; PP 92\%) and EAC 14 (ITT 73.3\%; PP 93.6\%) (ITT $p=0.67$; PP $p=0.76$ ). Poor compliance was reported in one patient in the EAC 14 group. The incidence of adverse events was comparable in the two groups.

Conclusion. One-week and 2-week triple treatments for $H$. pylori eradication are similar in terms of efficacy, safety and patient compliance.

S Afr Med J 2012;102(6):368-371.
Helicobacter pylori was first isolated by Warren and Marshall in 1982. It is a Gram-negative bacterium found on the gastric epithelium and induces chronic inflammation of the underlying mucosa. H. pylori infection is usually contracted in the first few years of life and tends to persist indefinitely unless treated. It is the most common cause of chronic gastritis and peptic ulcer disease and a risk factor for gastric adenocarcinoma and mucosa-associated lymphoid tissue lymphoma. First-line eradication triple therapy comprises a proton pump inhibitor (PPI) and two of the following antibiotics: clarithromycin, metronidazole and amoxicillin. ${ }^{1,2}$

However, consensus on the length of treatment is lacking. European practitioners considered 7 days of treatment sufficient to achieve a high eradication rate, in contrast to the USA, where a more consistent eradication rate was obtained by prolonging the duration of therapy to 14 days. ${ }^{1,2}$ Antibiotic resistance is a major cause of treatment failure. ${ }^{3}$ The prevalence of antimicrobial resistance in $H$. pylori varies regionally within and between countries. Increasing the duration of treatment or providing alternative antibiotics based on local resistance rates may improve eradication rates. ${ }^{4}$ Widespread and indiscriminate use of antibiotics in developing countries has resulted in a higher prevalence of resistance than in industrialised countries. ${ }^{5}$

The Maastricht III Consensus Guidelines state that effective eradication treatment should be successful in more than $80 \%$ of intention-to-treat (ITT) and $90 \%$ of per protocol (PP) treated patients, and that local studies should be done to establish the duration of treatment and eradication rates. ${ }^{1}$ In Kenya the first-line

Department of Medicine, Aga Khan University Hospital, Nairobi, Kenya Ahmed Sokwala, MD, MMed

Mahesh V Shah, MB ChB, MMed

Smita Devani, MB ChB, MRCP

Gerald Yonga, MB ChB, MMed, Dip Cardiol, MBA, FESC, FACC treatment is a PPI, amoxicillin and clarithromycin, but the optimal duration of therapy remains controversial. As we are not aware of local published studies to establish the rate of $H$. pylori eradication with the triple therapy and with different treatment periods, we decided to determine the optimum duration of triple therapy for $H$. pylori eradication.

\section{Methods}

All patients who presented between December 2009 and May 2010 with dyspepsia and who were positive for $H$. pylori on stool antigen tests were enrolled. Exclusion criteria were: previous attempts at H. pylori eradication, intake of bismuth, $\mathrm{H}_{2}$-antagonists, PPIs, or antibiotics within the 3 months prior to entry to the study, a history of intolerance and/or allergy to the study drugs, pregnancy or lactation, and alarm features. Alarm features were defined as age $>45$, gastrointestinal bleeding, anaemia, early satiety, unexplained weight loss, progressive dysphagia, odynophagia, recurrent vomiting, a family history of gastrointestinal cancer, and previous oesophagogastric malignancy. ${ }^{2}$ The study was approved by the local Ethics Committee and informed, written consent was obtained from each patient.

This was a prospective randomised investigator-blind comparative trial of 7- and 14-day triple therapy for H. pylori eradication conducted at the Aga Khan University Hospital, Nairobi. All patients who presented with dyspepsia and who were $H$. pylori-positive on stool antigen testing were randomised by simple computergenerated randomisation to receive either 7-day or 14-day triple therapy (esomeprazole $20 \mathrm{mg}$ twice a day, amoxicillin $1 \mathrm{~g}$ twice a day, clarithromycin $500 \mathrm{mg}$ twice a day). After 2 weeks the patients were reviewed by the principal investigator for assessment of sideeffects and compliance. A stool antigen test was repeated 6 weeks after the start of therapy to confirm eradication of $H$. pylori.

The patients were not charged for reviews at 2 and 6 weeks and a free $H$. pylori stool antigen test was performed to confirm eradication. Patients who still had H. pylori at 6 weeks were referred to the gastroenterology clinic for second-line therapy.

Hypothesising a difference of $20 \%$ between eradication rates achieved by the two regimens, and fixing a statistical power of at least 
$80 \%$ with a value of $\alpha<0.05$, we calculated the minimal number of patients to be included in each group ( 1 week or 2 weeks) to be 46 . With an estimated drop-out rate of $30 \%$, we then needed 120 patients in total and 60 patients in each group.

Compliance was assessed by counting the pills returned by the patients and was considered adequate with an intake $>90 \%$ of the drugs prescribed. The type and severity of adverse events during treatment were reported by patients on diary cards, to be returned no later than 1 week after ending therapy. Adverse effects were defined as mild when not interfering with normal daily activity; moderate when frequently interfering but allowing treatment to be completed, and severe when withdrawal was necessary. ${ }^{6}$

Data were analysed using SPSS version 15.0. Analysis of baseline characteristics and eradication success between different treatment protocols were performed using the chi-square test. The $t$-test was used to analyse the age distribution between the patient groups. Data were analysed according to ITT and PP criteria. The ITT analysis included all patients randomised to a treatment group, whereas in the PP analysis patients lost during follow-up or showing low compliance were excluded. The incidence and severity of adverse events in the two groups were also compared using the chi-square test.

\section{Results}

During December 2009 and May 2010, 146 patients with H. pyloripositive dyspepsia were identified. Of these, 26 were excluded: 21 did not meet inclusion criteria and 5 did not give consent. Of the 21,3 were pregnant, 4 were breastfeeding, 3 were allergic to penicillin, 6 had used PPIs within 4 weeks of presentation, and 4 had alarm features. The patients with alarm features were booked for endoscopy and follow-up in the gastroenterology clinic. Patients who did not give consent were given appropriate standard therapy. Remaining for enrolment and randomisation were 120 patients; 60 were randomised to receive esomeprazole $20 \mathrm{mg}$ twice daily in combination with amoxicillin $1 \mathrm{~g}$ twice daily and clarithromycin $500 \mathrm{mg}$ twice daily for 1 week (EAC 7) and 60 the same regimen for 2 weeks (EAC 14).

In the 7-day group 10 patients were lost to follow-up; 3 had travelled out of the city, 2 could not be reached by telephone and 5 did not see the benefit of coming back as they were asymptomatic. All the patients in the 7-day group were $>90 \%$ compliant (Fig. 1).

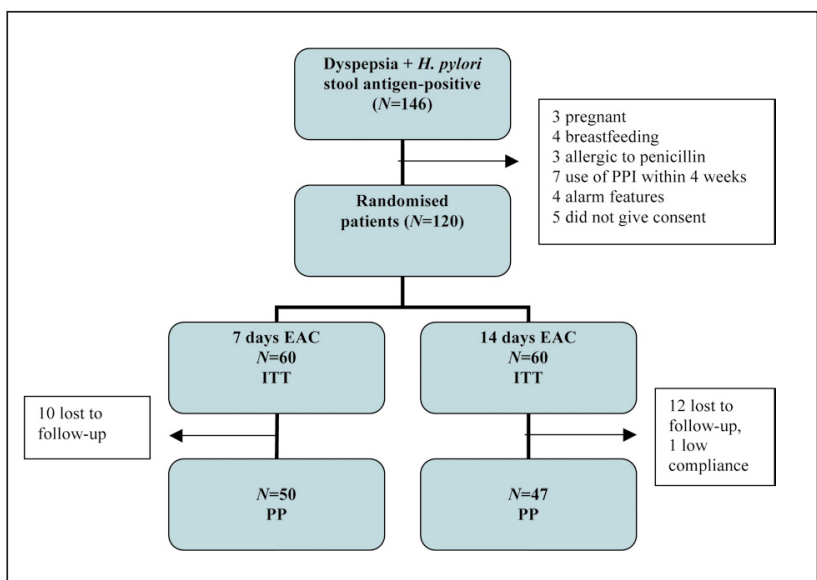

Fig. 1. Flow of participants through each phase of the study and incidence of patients lost to follow-up and with low compliance. $P P I=$ proton pump inhibitor; $I T T=$ intention to treat; $P P=$ per protocol; $E A C 7=$ esomeprazole $20 \mathrm{mg}$, amoxicillin $1 \mathrm{~g}$ and clarithromycin $500 \mathrm{mg}$ twice daily for 7 days; EAC 14 = esomeprazole $20 \mathrm{mg}$, amoxicillin $1 \mathrm{~g}$ and clarithromycin $500 \mathrm{mg}$ twice daily for 14 days.
In the 14-day group 12 patients were lost to follow-up; 4 had travelled out of the city, 2 could not be reached by telephone and 6 did not see the benefit of coming back as they were asymptomatic. One patient in the 14-day group was not compliant.

All patients were considered for ITT analysis, but only 97 for PP analysis, 23 patients (19.2\%) having being excluded because they had been lost to follow-up ( $n=22)$ or showed poor compliance $(n=1)$.

The mean age was in the fourth decade with no statistically significant difference between the two groups, and men and women were equally represented. Homogeneity in the basic demographic data allowed the two groups to be comparable.

Only 7 patients (5.8\%) were smokers and $43(35.8 \%)$ had a history of alcohol consumption. The difference in distribution of participants between the EAC 7 and EAC 14 groups was not significant for either smoking or alcohol consumption, with $p$-values of 0.24 and 0.85 respectively. Smoking is an independent risk factor for $H$. pylori treatment failure. ${ }^{7}$ In contrast, alcohol consumption may facilitate elimination of $H$. pylori infection among adults. ${ }^{8}$

All patients who had dyspepsia and were referred for endoscopy had $H$. pylori-positive gastritis. Patients with histology-proven $H$. pylori-positive gastritis were equally distributed between the two groups (Table 1).

Compliance was good; 97 of the participants $(98.98 \%)$ had a compliance of $>90 \%$ and only $1(1.02 \%)$ had $<90 \%$ compliance. However, 22 (18.3\%) were lost to follow-up. The only patient who had less than $90 \%$ compliance was in the EAC 14 group. We postulated that compliance was good because all patients were informed about the importance of $H$. pylori eradication and the possible drug sideeffects before commencing therapy. Bacterial resistance and poor patient compliance are believed to be the primary factors in $H$. pylori treatment failure. The occurrence of side-effects can reduce the compliance with treatment regimens and lead to the development of bacterial resistance. ${ }^{9}$

In the EAC 7 group 10 patients (16.7\%) were lost to follow-up whereas in the EAC 14 group 12 (20\%) were lost to follow-up, but

Table 1. Baseline characteristics of study patients

\begin{tabular}{llll}
\hline & EAC 7 (N=60) & EAC 14 (N=60) & $p$-value \\
\hline Gender & & & \\
$\quad$ Male & $25(41.7 \%)$ & $24(40 \%)$ & 0.85 \\
$\quad$ Female & $35(58.3 \%)$ & $36(60 \%)$ & \\
Age (yrs) mean & $34(10)$ & $36(11)$ & 1.0 \\
$\begin{array}{l}\text { (SD) } \\
\text { Smoking }\end{array}$ & & & \\
$\quad$ Yes & $5(8.3 \%)$ & $2(3.3 \%)$ & 0.24 \\
No & $55(91.7 \%)$ & $58(96.7 \%)$ & \\
Alcohol & & & \\
Yes & $21(35 \%)$ & $22(36.7 \%)$ & 0.85 \\
$\quad$ No & $39(65 \%)$ & $38(63.3 \%)$ & \\
Endoscopy & & & \\
Not done & $49(81.7 \%)$ & $47(78.3 \%)$ & 0.65 \\
$\quad$ Gastritis & $11(18.3 \%)$ & $13(21.7 \%)$ & \\
Compliance & & & \\
$>90 \%$ & $50(100 \%)$ & $47(97.9 \%)$ & 0.53 \\
$<90 \%$ & 0 & $1(2.1 \%)$ & 0.24 \\
Lost to follow-up & $10(16.7 \%)$ & $12(20 \%)$ & \\
& &
\end{tabular}


again the difference is not significant $(p=0.24)$. Most of the patients who did not come for follow-up visits felt better after the treatment.

\section{Eradication rates}

Our objective was to determine and compare the H. pylori eradication rates between 7- and 14-day triple therapy regimens according to the ITT and the PP analysis. All patients were considered for ITT analysis, but only 97 for PP analysis. The 23 patients (19.2\%) excluded were lost to follow-up $(n=22)$ or showed poor compliance $(n=1)$. On ITT analysis EAC 7 eradication rates were $76.7 \%$ (95\% confidence interval (CI) 66 - 87.4\%) whereas for EAC 14 the eradication rates were $73.3 \%$ (95\% CI $62.1-84.5 \%)$. The difference between the two groups was not statistically significant $(p=0.67$ ).

On PP analysis, the eradication rate in the EAC 7 group was $92 \%$ (95\% CI 84.5 - 99.5\%), which was slightly lower than the $93.6 \%$ achieved in the EAC 14 group (95\% CI 86.6 - 100\%), but was not significant $(p=0.76)$

There was no significant difference between EAC 7 and EAC 14 regimens in either the ITT $(p=0.67)$ or the PP $(p=0.76)$ analysis. The difference in eradication rates between the EAC 7 and EAC 14 groups was $-3.4 \%$ in the ITT analysis and $1.6 \%$ in the PP analysis (Table 2).

Gender, smoking habit, alcohol consumption and prior endoscopy had no influence on eradication rates, but our study was not powered for this conclusion. These factors were equally distributed in both groups and therefore unlikely to have significantly influenced the results.

\section{Compliance and tolerability}

Compliance was analysed in 98 patients, after exclusion of the 22 patients lost to follow-up. Compliance was found to be inadequate (intake of $<90 \%$ of total tablets) in 1 patient $(1.02 \%)$. Compliance was good in 97 (98.98\%) of the participants. However, 22 (18.3\%) were lost to follow-up. Compliance was comparable in both groups $100 \%$ in the EAC 7 group and $97.9 \%$ in the EAC 14 group ( $p=0.53$ ). The only patient who had $<90 \%$ compliance was in the EAC 14 group. Ten patients in the EAC 7 group (16.7\%) were lost to follow-up whereas 12 patients (20\%) in the EAC 14 group were lost to follow-up $(p=0.24)$.

Possible adverse effects of the regimen were headache, nausea, vomiting, diarrhoea, loss of appetite, taste disturbance, abdominal pain and rash. Taste disturbance was the commonest adverse effect; 17 (34\%) were affected in EAC 7 group and 25 (53.2\%) in the EAC 14

Table 2. H. pylori eradication rates calculated according to ITT and PP analysis

\begin{tabular}{llll}
\hline Regimen & EAC 7 & EAC 14 & p-value \\
\hline No. of patients & 60 & 60 & \\
Lack of compliance & 0 & 1 & \\
Lost to follow-up & 10 & 12 & \\
H. pylori eradicated & 46 & 44 & \\
ITT analysis $(n)$ & $46 / 60$ & $44 / 60$ & \\
\% eradicated & $76.7 \%$ & $73.3 \%$ & 0.67 \\
(95\% CI) & $(66-87.4)$ & $(62.1-84.5)$ & \\
PP analysis $(n)$ & $46 / 50$ & $44 / 47$ & \\
\% eradicated & $92 \%$ & $93.6 \%$ & 0.76 \\
(95\% CI) & $(84.5-99.5)$ & $(86.6-100)$ &
\end{tabular}

ITT = intention to treat; $\mathrm{PP}=$ per protocol; $\mathrm{EAC} 7=$ esomeprazole $20 \mathrm{mg}$, amoxicillin $1 \mathrm{~g}$ and clarithromycin $500 \mathrm{mg}$ twice daily for 7 days; EAC $14=$ = esomeprazole $20 \mathrm{mg}$, amoxicillin $1 \mathrm{~g}$ and clarithromycin $500 \mathrm{mg}$ twice daily for 14 days. group ( $p=0.057)$. All adverse events were mild and did not necessitate withdrawal or interfere with therapy. There was no statistically significant difference in the experience of side-effects between the treatment groups.

\section{Discussion}

International guidelines have allowed consensus on the best management and improved eradication rates of H. pylori. Therapy regimens and their duration could not be standardised because clinical data and efficacy of therapy vary between countries. This variation in the results could be due to factors such as bacterial virulence, environmental factors and widespread use of antibiotics. Increasing antimicrobial resistance has resulted in falling eradication rates with standard therapies. ${ }^{10}$ The first-line treatment remains clarithromycin, amoxicillin or metronidazole and a PPI twice daily, but recent studies show low eradication rates, and increased duration of therapy has been recommended to overcome this. There have been conflicting findings on the benefits of extending the length of traditional therapy. ${ }^{2}$ European guidelines indicate that triple therapy for 1 week is acceptable whereas, in the USA, 10 - 14 days of treatment is preferred. ${ }^{1,2}$ Regional antimicrobial resistance and eradication rates should determine the best treatment for H. pylori. ${ }^{1}$

No data or consensus about the duration of treatment for H. pylori in Kenya were available. Therefore we evaluated the triple therapy of esomeprazole $20 \mathrm{mg}$ twice a day, amoxicillin $1 \mathrm{~g}$ twice a day and clarithromycin $500 \mathrm{mg}$ twice a day in the first randomised controlled study comparing the efficacy of 1 and 2 weeks of triple therapy for H. pylori eradication in patients in Kenya. We found that 1 week of treatment with esomeprazole, clarithromycin and amoxicillin achieved $H$. pylori eradication rates of $76.7 \%$ and $92 \%$ by ITT and PP analyses, respectively. Extending the treatment to 2 weeks does not enhance eradication rates ( $73.3 \%$ and $93.6 \%$ by ITT and PP analyses, respectively). The difference between eradication rates achieved with 1 and 2 weeks of triple therapy is not statistically significant.

Studies of PPI-based triple therapy for 7 days, mainly from European countries, confirm that this remains a valid duration for H. pylori treatment. ${ }^{11}$ Our results are consistent with recent studies such as the HYPER study ${ }^{12}$ from Italy, which was a large, multicentre, double-blind and randomised study comparing the efficacy of 1-and 2-week regimens of omeprazole, amoxicillin and clarithromycin. Their ITT $(N=907)$ and PP $(N=661)$ analyses showed no significant differences between the eradication rates at 1 week (ITT 79.7\%; PP $83.6 \%$ ) and 2 weeks (ITT 81.7\%; PP 84.9\%) (ITT $p=0.53$; PP $p=0.71$ ). Laine et al. ${ }^{13}$ reported that ITT eradication percentages with triple therapy (omeprazole, amoxicillin and clarithromycin) were $86 \%$ at 7 days, $90 \%$ at 10 days and $92 \%$ at 14 days in a study at a single institution. A multicentre study in 11 centres in Asia and Africa, where patients with endoscopy-proven duodenal ulcer and who were $H$. pylori-positive were treated with clarithromycin $500 \mathrm{mg}$, omeprazole $20 \mathrm{mg}$, and amoxicillin $1000 \mathrm{mg}$, all given twice daily for 7 days, recruited a total of 117 patients. Overall, $H$. pylori eradication rates were $85 \%$ by PP analysis and $80 \%$ by ITT analysis. Only 25 patients were from South Africa, where eradication rates were $86 \%$ (19/22) (95\% CI 65.1 - 97.1\%) by PP analysis and 76\% (19/25) (95\% CI $54.9-90.6 \%)$ by ITT analysis. ${ }^{14}$ These results are in keeping with our study.

Clinicians must stress the importance of taking the medications as prescribed to minimise the likelihood of antibiotic resistance developing, as this is a major cause of treatment failure. The prevalence of antimicrobial resistance in $H$. pylori shows regional variation within and between countries. Clarithromycin resistance 
varies significantly, ranging from close to zero up to $25 \%$ and has significant impact on eradication success, but bacterial resistance to amoxicillin is $<1 \%$ in most countries. ${ }^{1}$ Alternative antibiotics based on local resistance rates may improve eradication rates. The widespread and indiscriminate use of antibiotics in developing countries has resulted in a higher prevalence of resistance than in industrialised countries. ${ }^{5}$

One week of triple therapy remains the recommended first line treatment for $H$. pylori eradication ${ }^{12}$ in regions where primary resistances of clarithromycin or metronidazole are lower than 15 $20 \%$ or $40 \%$, respectively.

Data on $H$. pylori antibiotic sensitivity profiles in Kenya are scarce, a recent study showing all $H$. pylori largely sensitive to clarithromycin (100\%), amoxicillin $(100 \%)$ and metronidazole (95.4\%). There was, however, occasional resistance to metronidazole (4.6\%). ${ }^{15}$ This confirms our finding that 7 -day triple therapy is adequate for $H$. pylori eradication because local resistance rates are very low.

No serious adverse events were documented in either treatment group. The adverse events were mild and did not necessitate withdrawal or interfere with therapy. There was no statistically significant difference in the side-effects between the treatment groups. The tolerability of triple therapy has been good and similar for 1 - and 2-week regimens. ${ }^{12}$

Compliance with antimicrobial therapy is better with shortduration therapy. This is particularly relevant with $H$. pylori eradication regimens because side-effects such as diarrhoea or taste disturbance can lead to discontinuation of therapy in some patients, with potential treatment failure. All our patients were educated on the importance of $H$. pylori eradication and possible side-effects, with the result that patient compliance was high.

In conclusion, this study shows that the efficacy and safety of 1 week of triple therapy including esomeprazole with amoxicillin and clarithromycin does not differ significantly from 2 weeks of treatment for the eradication of $H$. pylori.

\section{References}

1. Malfertheiner P, Megraud F, O'Morain C, Bazzoli F, El-Omar E, Graham D, et al. Current concepts in the management of Helicobacter pylori infection: the Maastricht III Consensus Report. Gut 2007;56(6):772-781. [http://dx.doi.org/10.1136/gut.2006.101634].

2. Chey WD, Wong BC. American College of Gastroenterology guideline on the management of Helicobacter pylori infection. Am J Gastroenterol 2007;102(8):1808-1825. [http://dx.doi.org/10.1111/ j.1572-0241.2007.01393.x].

3. Megraud F. $H$. pylori antibiotic resistance: prevalence, importance, and advances in testing. Gut 2004;53(9):1374-1384. [http://dx.doi.org/ 10.1136/gut.2003.022111].

4. Fischbach L, Evans EL. Meta-analysis: the effect of antibiotic resistance status on the efficacy of triple Fisch and quadruple first-line therapies for Helicobacter pylori. Aliment Pharmacol Ther 2007;26(3):343-357.

5. Gerrits MM, van Vliet $\mathrm{AH}$, Kuipers EJ, Kusters JG. Helicobacter pylori and antimicrobial resistance molecular mechanisms and clinical implications. Lancet Infect Dis 2006;6(11):699-709. [http://dx.doi. org/10.1016/\$1473-3099(06)70627-2].

6. de Boer WA, Thys JC, Borody TJ, Graham DY, O’Morain C, Tytgat GN. Proposal for use of standard side effect scoring system in studies exploring Helicobacter pylori treatment regimens. Eur Gastroenterol Hepatol 1996;8(7):641-643

7. Suzuki T, Matsuo K, Ito H, Sawaki A, Hirose K, Wakai K, et al. Smoking increases the treatment failure for Helicobacter pylori eradication. Am J Med 2006;119(3):217-224. [http://dx.doi.org/10.1016/]. amjmed.2005.10.003].

8. Murray LJ, Lane AJ, Harvey IM, Donovan JL, Nair P, Harvey RF. Inverse relationship between alcohol consumption and active Helicobacter pylori infection: the Bristol Helicobacter project. Am Gastroenterol 2002;97(11):2750-2755. [http://dx.doi.org/10.1016/S0002-9270(02)05481-3].

9. de Bortoli N, Leonardi G, Ciancia E, Merlo A, Bellini M, Costa F, et al. Helicobacter pylori eradication: a randomized prospective study of triple therapy versus triple therapy plus lactoferrin and probiotics. a randomized prospective study of triple therapy versus triple therapy plus lactoferrin and probio

10. Egan BJ, Katicic M, O'Connor HJ, O'Morain CA. Treatment of Helicobacter pylori. Helicobacter 2007;12 (suppl 1):31-37. [http://dx.doi.org/10.1111/j.1523-5378.2007.00538.x]

11. Megraud F. Update on therapeutic options for Helicobacter pylori-related diseases. Curr Infect Dis Rep 2005;7(2):115-1120.[http://dx.doi.org/10.1007/s1 1908-005-0071-4]

12. Zagari RM, Bianchi-Porro G, Fiocca R, Gasbarrini G, Roda E, Bazzoli F. Comparison of 1 and 2 week of omeprazole, amoxicillin and clarithromycin treatment for Helicobacter pylori eradication: the HYPER Study. Gut 2007;56(4):475-479. [http://dx.doi.org/10.1136/gut.2006.102269].

13. Laine L, Estrada R, Trujillo M, Fukanaga K, Neil G. Randomized comparison of differing periods of twice-a-day triple therapy for the eradication of Helicobacter pylori. Aliment Pharmacol Ther 1996;10(6):1029-33. [http://dx.doi.org/10.1046/j.1365-2036.1996.111282000.x].

14. Wong BC, Chang FY, Abid S, et al. Triple therapy with clarithromycin, omeprazole, and amoxicillin for eradication of Helicobacter pylori in duodenal ulcer patients in Asia and Africa Aliment Pharmacol Ther 2000;14(11):1529-1535. [http://dx.doi.org/10.1046/j.1365-2036.2000.00863.x].

15. Kimanga AN, Revathi G, Kariuki S, Sayed S, Devani S. Helicobacter pylori: prevalence and antibiotic susceptibility among Kenyans. S Afr Med J 2010;100(1):53-57. 